Technological University Dublin

DÜBLIN

ARROW@TU Dublin

2009-01-01

\title{
Simple Versatile Shearing Interferometer Suitable for Measurements on a Microscopic Scale
}

\author{
Emilia Mihaylova \\ Technological University Dublin, emilia.mihaylova@tudublin.ie \\ Vincent Toal \\ Technological University of Dublin, vincent.toal@tudublin.ie
}

Follow this and additional works at: https://arrow.tudublin.ie/cieoart

Part of the Engineering Commons, and the Optics Commons

\section{Recommended Citation}

Mihaylova, E. \&Toal, V. (2009) Simple versatile shearing interferometer suitable for measurements on a microscopic scale. Optics and Lasers in Engineering vol. 47, pp.271-273. doi:10.1016/

j.optlaseng.2008.05.003

This Article is brought to you for free and open access by the Centre for Industrial and Engineering Optics at ARROW@TU Dublin. It has been accepted for inclusion in Articles by an authorized administrator of ARROW@TU Dublin. For more information, please contact arrow.admin@tudublin.ie, aisling.coyne@tudublin.ie, gerard.connolly@tudublin.ie.

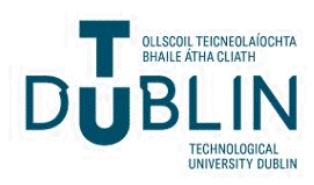




\title{
Simple Versatile Shearing Interferometer
}

\section{Suitable For Measurements on a Microscopic Scale}

\author{
Emilia Mihaylova*, Vincent Toal \\ Centre for Industrial and Engineering Optics, Dublin Institute of Technology, \\ Kevin Street, Dublin 8, Ireland \\ *Corresponding author: e-mail: emilia.mihaylova@dit.ie \\ tel: +35314024702; fax: + 35314024988
}

\begin{abstract}
Microelectromechanical systems (MEMS) behave differently from massive samples. Conventional testing and inspection techniques usually fail at the microscale. Recently there has been an increasing interest in the application of optical techniques for microstructure testing, because they are high-resolution, non-contact, full-field, fast and relatively inexpensive. New interferometric systems, which are suitable for microscopic optical metrology, are of interest for engineering and industrial applications.

A modified electronic speckle pattern shearing interferometer (ESPSI) with a very simple shearing device has been designed for metrology applications on the microscale. The shearing device consists of two partially reflective glass plates. The reflection coefficients of the coatings are 0.3 and 0.7 respectively. The distance and the tilt between the two glass plates control the size of the shear. A long working distance microscope objective is attached to the CCD camera to form a field of view variable over several millimetres in width. The suitability of the system for microscopic measurements is demonstrated. The capability of the system for phase-shifting is also demonstrated. The results obtained are promising for future applications of the ESPSI system for testing and characterisation of MEMS.
\end{abstract}

KEYWORDS: ESPSI, MEMS, shearing interferometry, microscopy, microcomponents 


\section{INTRODUCTION}

Developments in the field of semiconductors are leading to integrated circuits with three-dimensional features and moving parts, called micro-electromechanical systems (MEMS). Research and development in MEMS started in the early 1970's. Nowadays MEMS are moving rapidly towards commercialization and their mechanical characterization has become an issue of major importance. Recently there has been an increasing interest in the application of optical techniques for microstructure testing, because they are high-resolution, non-contact, full-field, fast and relatively inexpensive. White light interferometry is now a routine technique used for topographic measurements of MEMS [1]. Digital holography is also well suited for MEMS characterization: for three-dimensional imaging and for quantitative displacement and shape measurements [2]. However we are unaware of any publication that reports the development of electronic speckle pattern shearing interferometry (ESPSI) for MEMS testing or other investigations on a microscopic scale.

ESPSI enables direct measurements of displacement derivatives to be made with variable sensitivity [3, 4]. A common lateral shear interferometer uses a plane parallel glass plate as a shearing device. The amount of shear is selected by the thickness and tilt of the plate [5]. The disadvantages of this simple shearing interferometer are that the resulting interferogram has low fringe visibility and phase-shifting techniques cannot be applied. Phase-shifting shearography is more sensitive than simple shearography [6] and can provide three-dimensional (3D) plots of the displacement derivatives [3]. Time-averaged fringes can be obtained in shearography in the same way as in electronic speckle pattern interferometry (ESPI) [7]. An advantage of shearography is that it is less sensitive to object instability and turbulence than ESPI because two laterally sheared images interfere with each other so the interferometer is nearly a common path one. In addition, the sensitivity of the shearographic system is easy to adjust by changing the amount of shear introduced between the two images.

We present an upgraded version of a simple ESPSI system previously reported by us [8]. The novelty of the system reported here is the introduction of a long working distance microscope objective in front of the CCD camera to form a field of view variable over several millimeters in width. We demonstrate that the system is capable of qualitative and quantitative measurements. 


\section{THEORY}

The theory of the phase-shifting shearography applicable to our measurements was presented previously [8]. In our case (Fig. 1) the illuminating object beam lies in the $(x, z)$ plane and there is no sensitivity to displacement along the y axis so long as the beam is well collimated. The system is suitable for implementing digital phase shifting to provide fringe analysis. There are a number of algorithms available for the purpose. The algorithm we use is a five bucket type in which four interferograms are captured having successive phase shifts of $\pi / 2, \pi, 3 \pi / 2$. A fifth interferogram with zero phase shift is captured after the loading is applied and the corresponding phase map is obtained from these data [9].

\section{EXPERIMENT}

The experimental arrangement of the simple microscopic ESPSI system with two partially reflective glass plates is presented in Figure 1.

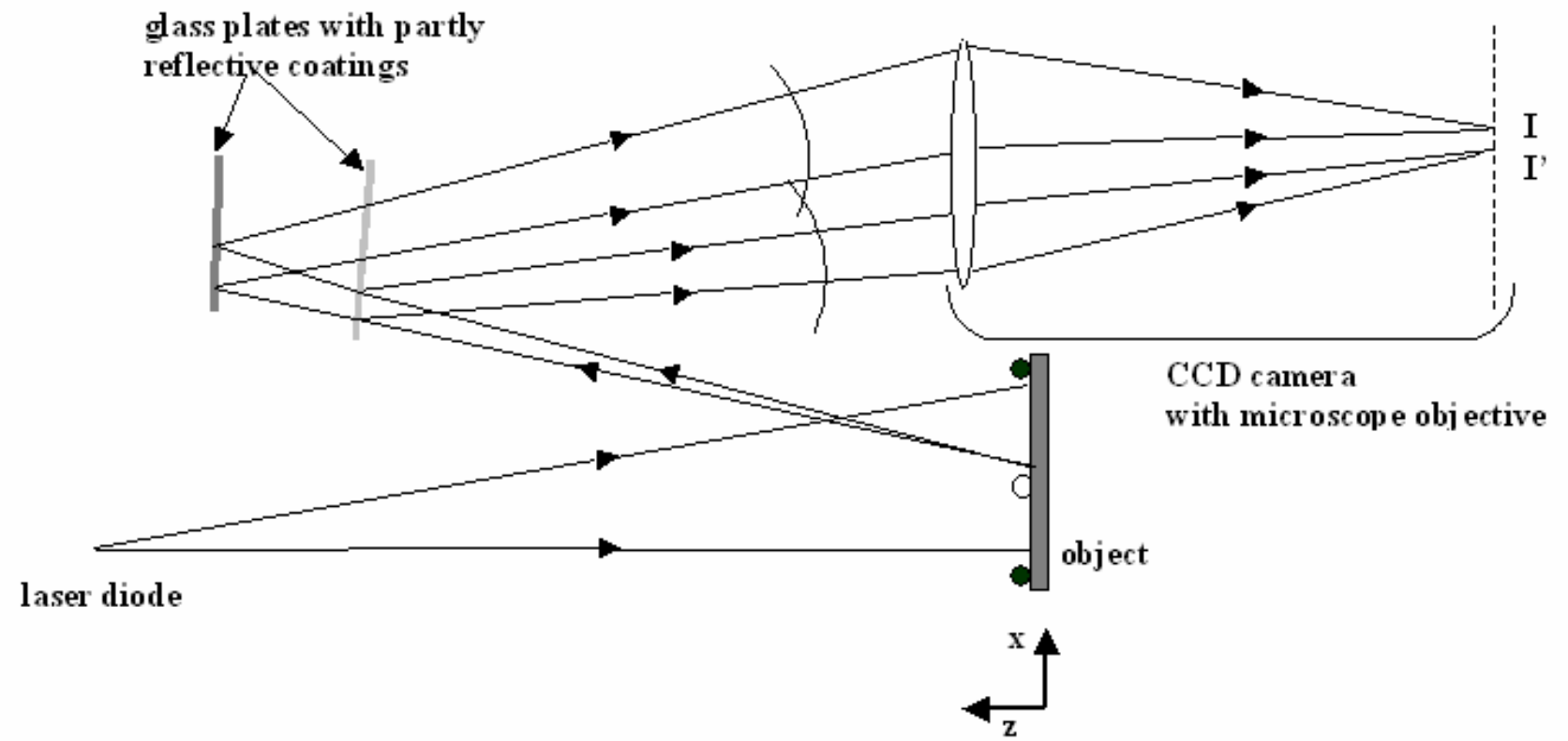

Fig. 1. Experimental set-up of the microscopic ESPSI system with two glass plates as shearing device. 
A He-Ne laser $(\lambda=633 \mathrm{~nm}$ ) is used as the light source. A laser beam illuminates the object at an angle to the normal to the object surface so that the system is sensitive to displacement in both $\mathrm{x}$ and $\mathrm{z}$ directions. The reflection coefficients of the coatings are 0.3 for the first glass plate and 0.7 for the second glass plate. In this way the two sheared images have approximately the same intensity and the fringe contrast is very good. The interference patterns are recorded by a CCD camera, equipped with a long working distance Mitutoyo microscope objective (10x), and stored in a computer.

The proposed ESPSI system was used in studying mechanical deformations. The classical subtraction method was used for detection of the deformations. Replacing the microscopic objective with a conventional TV lens makes the system suitable for macroscopic studies. It is demonstrated that the proposed system is capable of implementing digital phase shifting.

\section{RESULTS AND DISCUSSION}

The first set of experiments was directed towards detection of deformations on microscale. Highly flexible amorphous material (blue glue) was used to show the ESPSI fringes due to relaxation processes. Results are shown in Fig. 2. Artificial defects were made on the surface of the material using a metal bar. Following deformation, ESPSI fringes were observed in real time. The observed fringe pattern called a shearogram depicts displacement derivatives and thus contains the strain information and can be evaluated numerically. The obtained shearograms are of good quality and show repeatability.

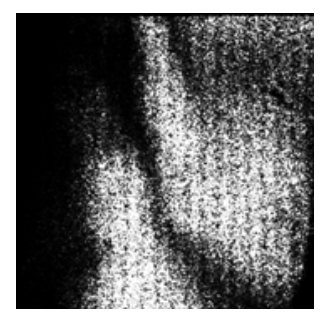

a

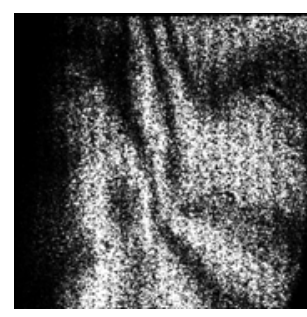

b

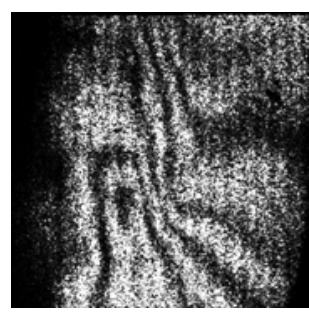

C

Fig. 2. ESPSI fringes in blue glue: a) $30 \mathrm{~s}$ after deformation; b) $33 \mathrm{~s}$ after deformation; c) $36 \mathrm{~s}$ after deformation. The shear is $0.5 \mathrm{~mm}$. The field of view is $2 \mathrm{~mm} \times 2 \mathrm{~mm}$. 
The high sensitivity of the microscopic ESPSI system is shown in that way. It is able to detect time dependant strain changes in viscoelastic materials due to molecular rearrangement. It could be used for strain studies in MEMS. In addition it will be useful for strain investigations in different elastic materials such as biopolymers, and micro-mechanical systems made from these materials.

During a second set of experiments the system was configured in phase-shifting mode. The ESPSI system was used with a Fujinon TV lens HF25HA-1, replacing the microscope objective. One of the glass plates was mounted on a piezoelectric transducer (PZT). This phase-shifter allows the introduction of a known phase shift. The consecutive speckle shearing interference patterns are recorded by a CCD camera and stored in a computer. The phase difference (after subtraction) is displayed as a phase map. The phase-shifting ESPSI system is controlled remotely by software [10].

We used a bending test of a fibreglass reinforced plastic (FRP) beam to demonstrate the phase-shifting ESPSI system with two glass plates. One end of the beam was clamped, the other end was simply supported. Figure 3 presents results obtained from the phase-shifting ESPSI system. The phase at each point was evaluated [9, 10] from the phase data and the 3D plot of the phase distribution obtained. The phase map is indicative of both out-of-plane displacement gradient and in-plane displacement gradient because the illuminating beam is at an angle to the normal.

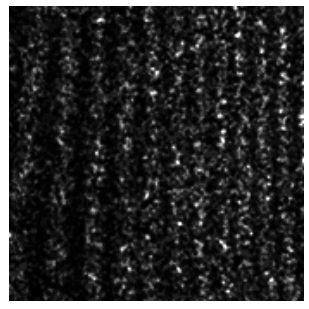

a

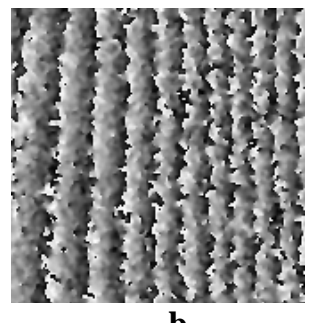

b

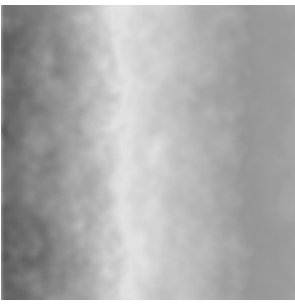

C

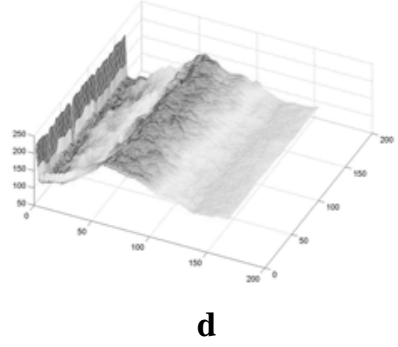

d

Fig. 3. ESPSI fringes on FRP beam, recorded with ESPSI system with two glass plates under deflection of $40 \mu \mathrm{m}$ : a) ESPSI fringes; b) wrapped phase map; c) unwrapped phase map; d) psedo-3D plot of the phase distribution. The field of view is $32 \mathrm{~mm} \times 32 \mathrm{~mm}$. 


\section{CONCLUSIONS}

A simple electronic speckle pattern shearing interferometer system suitable for measurements on a microscopic scale has been proposed. The incorporation of a long working distance microscopic objective allows investigation of microstructures at sufficient working distances for good illumination. The proposed ESPSI system is versatile and flexible and offers a simple way to introduce discrete shear steps between two images. It has been proved that the system is capable of detecting of mechanical deformations at microscale. The proposed system could be used in a phase-shifting mode for detailed fringe analysis. An additional advantage is the low cost of such a system. The results obtained are promising for future development and applications of this and similar ESPSI systems.

\section{REFERENCES}

1. S. Jorez, A. Cornet, J-P. Raskin, "MEMS profilometry by low coherence phase shifting interferometry: Effect of the light spectrum for high precision measurements”, Optics Comm. 263, 6-11 (2006).

2. L. Xu, X. Peng, J. Miao, and A. K. Asundi, "Studies of digital microscopic holography with applications to microstructure testing”, Appl. Opt. 40 (28), 5046-5051 (2001).

3. W. Steinchen and L. Yang, "Digital shearography: theory and application of digital speckle pattern shearing interferometry", SPIE, Washington (2003).

4. R. S. Sirohi, "Speckle methods in experimental mechanics", in Speckle Metrology, Ed. R. S. Sirohi, Mercel Dekker, New York (1993).

5. J. C. Wyant, “A simple interferometric MFT instrument”, Optics Comm. 19 (1), 120-121 (1976).

6. M. Owner-Petersen, "Decorrelation and fringe visibility: on the limiting behaviour of various electronic speckle pattern correlation interferometers”, J. Opt. Soc. Am. 8, 1082-1089 (1991).

7. Jones R, Wykes C., Holographic and speckle interferometry. Cambridge: Cambridge University Press (1989).

8. Emilia M. Mihaylova, Maurice P. Whelan, Vincent Toal, "A simple phase-shifting lateral shearing interferometer”, Optics Letters 29 (11), 1264, June 2004.

9. P. K. Rastogi, Digital Speckle Pattern Interferometry and Related Techniques (book), Ed. P. K. Rastogi, John Wiley \& Sons Ltd., New York (2001).

10. A. Langhoff and M. Whelan, "EspiTest Software”, Copyright @ 1998, European Commission, DG - Joint Research Centre, Ispra, Italy. 